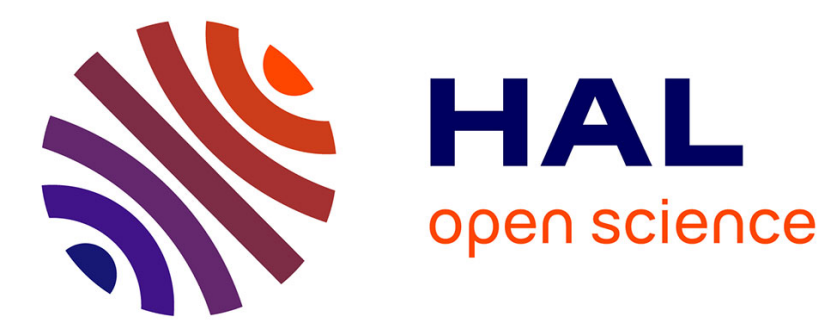

\title{
Modeling magnetostratigraphy in a borehole
}

Yves Gallet, Vincent Courtillot

\section{To cite this version:}

Yves Gallet, Vincent Courtillot. Modeling magnetostratigraphy in a borehole. Geophysics, 1989, 54 (8), pp.973-983. 10.1190/1.1442740 . insu-01863511

\section{HAL Id: insu-01863511 https://hal-insu.archives-ouvertes.fr/insu-01863511}

Submitted on 28 Aug 2018

HAL is a multi-disciplinary open access archive for the deposit and dissemination of scientific research documents, whether they are published or not. The documents may come from teaching and research institutions in France or abroad, or from public or private research centers.
L'archive ouverte pluridisciplinaire HAL, est destinée au dépôt et à la diffusion de documents scientifiques de niveau recherche, publiés ou non, émanant des établissements d'enseignement et de recherche français ou étrangers, des laboratoires publics ou privés. 


\title{
Modeling magnetostratigraphy in a borehole
}

\author{
Yves Gallet ${ }^{\star}$ and Vincent Courtillot ${ }^{\star}$
}

\begin{abstract}
The magnetic field along a hole bored through a sequence of dipping layers with varying magnetization and planar interfaces is calculated. The emphasis is on recovering remanent magnetization polarity transitions for magnetostratigraphic applications (dating, correlations), although intensity of remanent magnetization can in itself be a useful rock property. Results are presented for various cases of geologic interest in the form of axial vertical profiles and section maps of the holes at varying distances from polarity reversal interfaces. The vertical profiles demonstrate a resolving power of about six times the hole radius; meaningful magnetostratigraphies can be expected for rocks with a magnetization larger than $10^{-3} \mathrm{Am}^{-1}$, for instruments with a sensitivity of $0.1 \mathrm{nT}$. In a number of natural occurrences, it may not be necessary to resort to progressive demagnetization to recover the polarity sequence. Depending on which magnetic field and magnetization component one looks at, the section maps display characteristic patterns, in which, for instance, the direction of magnetization and the dip of the layers interfere. These maps are discussed in some detail. They can be used as guidelines to build a multisensor vector magnetometer (downhole magnetostratigraphic tool), whose output should be coupled with measurement of magnetic susceptibility for reduction of induced magnetization and with output from a surface instrument in a differential operation mode to reduce transient magnetic variations.
\end{abstract}

\section{INTRODUCTION}

Magnetostratigraphy, i.e., the determination of the sequence of polarities of the primary remanent magnetization in a stratigraphic section, is now well recognized as a potentially very powerful tool for correlation and absolute dating (e.g., Tauxe et al., 1987). In conventional magneto- stratigraphy, a large number of rock samples with as dense and continuous a distribution as possible are collected from an outcrop. The samples are oriented, brought back to the laboratory, and subjected to a set of progressive demagnetization techniques (alternating field, thermal, chemical) prior to measurement with a sensitive magnetometer, all of this in field-free space. These are all steps in a classical paleomagnetic study (e.g., McElhinny, 1973). It would be extremely useful (and far less time-consuming) to be able to obtain magnetostratigraphy directly from continuous downhole measurements, i.e., to develop a logging tool for additional correlation and age determination.

A sensitive magnetometer in a hole measures a magnetic field resulting from the vector superposition of several components (for the time variations see, e.g., Courtillot and Le Mouël, 1988). First is the main or principal field $\mathbf{B}_{p}$ which originates in the Earth's core and varies extremely slowly as a function of time (secular variation). The second field $\mathbf{B}_{t}$ is a sometimes rapidly varying field with its origin in electrical currents circulating above the upper atmosphere. Third, there is a static field $\mathbf{B}_{a}$ due to crustal sources, i.e., permanent and induced rock magnetization. The induced part itself is a function of the existing total field, which is usually dominated by $\mathbf{B}_{p}$ and is proportional to the rock magnetic susceptibility $\chi$ (assumed here to be isotropic). The remanent part of the static field is dominated by contributions from sources close to the instrument in the hole and includes the primary remanence acquired at the time of formation of the rock, plus possible later overprints.

Of interest are the direction, or at least the magnetic polarity of the primary remanence as a function of depth and correlations with the known geomagnetic reversal time scale in order to identify changes in depositional rate, gaps, and overall age of the rocks. To extract this information, we must solve a number of problems. Separating the static field of local origin from the sum $\mathbf{B}_{p}+\mathbf{B}_{t}$ can, in principle, be achieved by operating a separate but identical instrument close to the opening of the borehole and then calculating at each instant a difference vector with respect to the downhole measurement (e.g., Zlotnicki, 1986). The downhole magnetic susceptibility must be known, and with it the induced part of

Manuscript received by the Editor March 17, 1988; revised manuscript received December 12, 1988.

*Laboratoire de Paleomagnetisme, Institut de Physique de Globe du Paris, 4 place Jussieu, F-75252 Paris Cedex 05, France.

(c) 1989 Society of Exploration Geophysicists. All rights reserved. 
the field $\chi \mathbf{B}$. Magnetic susceptibility provides information on the amount of paramagnetic (and superparamagnetic and diamagnetic) minerals present in the bored layer (e.g., Rochette, 1988). An induction tool which measures $\chi$ (Clerc et al., $1983)$ is a necessary component of a magnetostratigraphic tool.

The remanent part of the downhole magnetic field can therefore be accessed. The next step would be to identify and erase any possible secondary magnetic overprint. No method able to achieve this has yet been proposed, but we will assume in a first approach that secondary remagnetizations can be neglected (see the Discussion section). Fortunately, secondary components are often absent or weak enough not to hide the magnetic polarity of the primary component. In that case, the magnetostratigraphic reversal sequence can be recovered successfully.

The development of high-sensitivity downhole magnetometers requires a precise formulation and solution to the forward problem of calculating the magnetic field distribution due to layered rocks with configurations of geologic interest (e.g., Parker and Daniell, 1979). We propose here a complete, detailed solution to the horizontal-layer case (see also Pozzi et al., 1988). We next present an approach to the case of dipping (parallel or intersecting) layers. The results are presented in terms of field maps in the hole at various distances from a reversal interface. The influences of remanence direction and dip of the interface are evaluated, using a matrix-map representation of the different contributions $C_{i j}$ (corresponding to a given field component $\boldsymbol{B}_{\boldsymbol{i}}$ and magnetization $J_{j}$ ). Vertical profiles along the hole axis are calculated to investigate the accuracy of locating the position of the interface from such a profile. Finally, these results can be used to constrain the geometry and the sensitivity of a downhole magnetometer and provide synthetic examples of the potential results that could be obtained in a hole. The direct analysis presented here could also serve as a basis for solving the inverse problem of determining rock magnetization from magnetic measurements in a real (noisy) case.

\section{MODELING}

\section{The model}

The borehole, assumed to be a vertical circular cylinder of radius $R$, penetrates a layered medium, in which each layer has a uniform magnetization. Three cases are considered: (1) all layers are horizontal; (2) all layers are parallel but have a nonzero dip (this is equivalent to an inclined borehole penetrating horizontal layers, except close to the surface); and (3) the interfaces between layers have different dips.

The property of linear superposition of magnetostatic fields allows one to determine the magnetic field due to the bored layers as the sum of the fields generated by each (nonbored) layer (index $k$, magnetization $J_{k}$ ) and by cylindrical segments with reversed magnetization $-J_{k}$ (defined as the part of the hole bounded by the bored interfaces of layer $k$ ). The magnetic field for each layer may be calculated by replacing each uniformly magnetized body with a fictitious (equivalent) monopole surface distribution with surface den- sity $d q=\mathbf{J} \cdot \mathbf{n} d S$, where $d S$ is the surface element with outward unit normal $\mathbf{n}$ ( $J$ is in $\mathrm{Am}^{-1}$ and $d q$ in Am).

Because of the dimensions of the hole, we can safely use a flat-earth approximation and assume that layers have infinite lateral extent. For a single, uniformly magnetized layer of uniform thickness, the magnetic induction (which, as is often done, we abusively call the "magnetic field") is zero everywhere outside the layer and is normal to the layer inside with magnitude $B=-\mu_{0} \mathbf{J} \cdot \mathbf{n}=-\mu_{0} J_{n}$, where $\mu_{0}$ is the magnetic permeability of free space $4 \pi 10^{-7} \mathrm{Hm}^{-1}$ and $B$ is in teslas. For a single charged plate (layer boundary), the field is everywhere normal and equal to $\left(\mu_{0} / 2\right) \mathbf{J} \cdot \mathbf{n} \operatorname{sgn}(z)$, where sgn is the sign function and $z$ is in the sense of the chosen $\mathbf{n}$. When the layer dips are different, i.e., when layer thickness is not uniform, we must allow for the intersections of charged plates at finite distances (intersections of layer boundaries with the earth surface in the second model, intersection of all layers in the third model). The forward problem can always be fully solved but soon becomes too complex for practical purposes. We assume that the dips are small enough that the intersections can safely be ignored; this is discussed below.

The superposition property can be used to model the finite cylinders as the superposition of two semiinfinite cylinders with opposite magnetization. The magnetic field generated by each semiinfinite cylinder can be treated as the sum of the fields of surface charges on two surfaces (Figure 1): the lateral flanges of the cylinder (which we call shape 1), where surface charge involves only the horizontal components of magnetization, and the upper ellipse (shape 2), which of course becomes a circle for zero dip. The field at point $\mathrm{P}$ derives from a potential $V(P)$, which is the integral of $d V(P)=\left(\mu_{0} / 4 \pi\right) \mathbf{J} \cdot \mathbf{n} d S / r$ over each surface or shape, $r$ being the distance from $P$ to the generating point $Q$ of the surfaces (Figure 1). We have chosen a Cartesian coordinate system in which $\mathrm{Oz}$ is the downward oriented axis of the hole and $\mathrm{Ox}$ (Figure 1b) the downdip (i.e., long axis of the ellipse) direction. The field components $B_{i}(i=x, y, z)$ are linearly related to the uniform magnetization components $J_{j}$ through a second-order symmetric tensor $C_{i j}$. $C_{i j}$ and $B_{i}$ are calculated in Appendix A for shape 1 and in Appendix B for shape 2 when the top of the semiinfinite cylinder is dipping. The double integral, which defines each coefficient $C_{i j}$, can be reduced analytically to a number of simple integrals with elliptical kernels. They are summarized for convenience in Table 1. The integrals have been calculated numerically using Simpson's method, either in Cartesian or in polar coordinates, with a sampling interval of $0.002 R$ for lengths and $2^{\circ}$ for angles. They are combined in the next section to generate the full field maps and profiles for the cases we are interested in.

\section{Numerical results}

Horizontal layers.-In order to display as clearly as possible the magnetic signature of a polarity transition, we first analyze the simplest nontrivial configuration in which a $10 R$ thick layer with zero magnetization overlies a substratum with uniform magnetization $\mathbf{J}$; the layers are assumed to be flat. The field values along the axis of the hole reduce to 

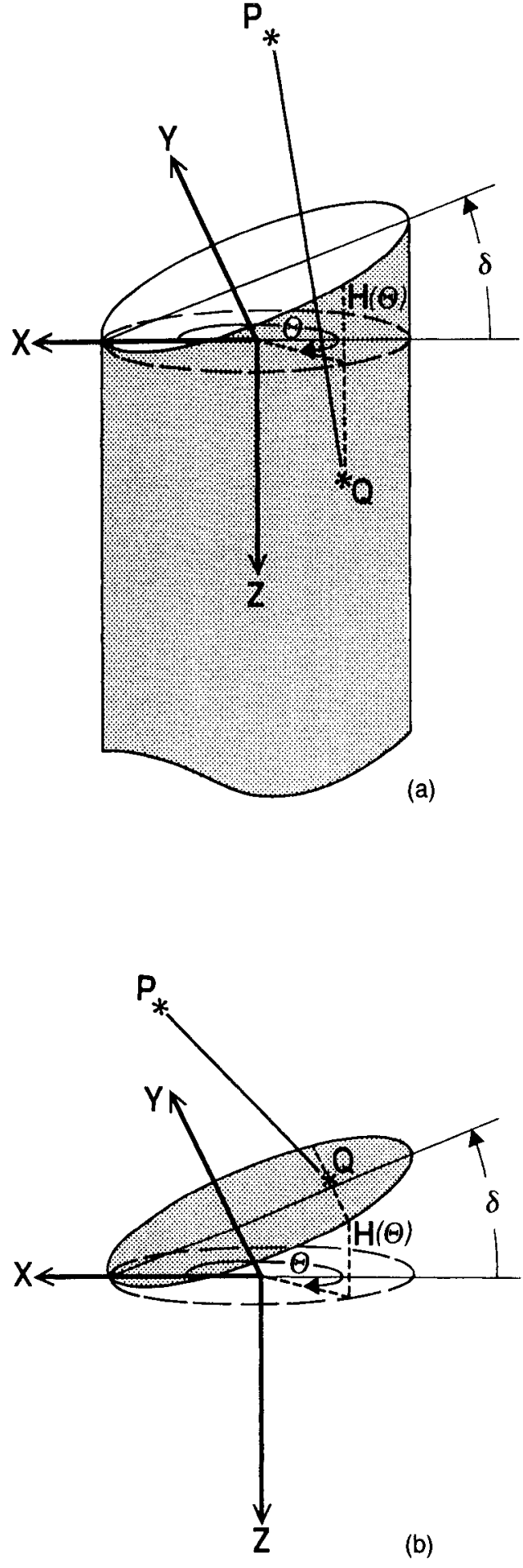

FIG. 1. The geometry used for calculating the magnetostatic field components in a borehole: (a) the lateral surface of a semiinfinite cylinder (shape 1), (b) the upper ellipse, inclined at angle $\delta$ (shape 2).
Table 1. The nine coefficients of the matrix $C_{i j}$ for a semiinfinite cylinder.

$$
\begin{aligned}
& C_{x x}=\frac{\mu_{0}}{4 \pi} R \int_{0}^{2 \pi} A(\Theta ; x, y, z)(x-R \cos \Theta) \cos \Theta d \Theta \\
& +\frac{\mu_{0}}{4 \pi} \tan \delta \int_{-R}^{R} K(\xi ; x, y, z)(x-\xi) d \xi \\
& C_{x y}=\frac{\mu_{0}}{4 \pi} R \int_{0}^{2 \pi} A(\Theta ; x, y, z)(x-R \cos \Theta) \sin \Theta d \Theta \\
& C_{x z}=\frac{\mu_{0}}{4 \pi} \int_{-R}^{R} K(\xi ; x, y, z)(x-\xi) d \xi \\
& C_{y x}=\frac{\mu_{0}}{4 \pi} R \int_{0}^{2 \pi} A(\Theta ; x, y, z)(y-R \sin \Theta) \cos \Theta d \Theta \\
& +\frac{\mu_{0}}{4 \pi} \tan \delta \int_{-R}^{R} L(\xi ; x, y, z) d \xi \\
& C_{y y}=\frac{\mu_{0}}{4 \pi} R \int_{0}^{2 \pi} A(\Theta ; x, y, z)(y-R \sin \Theta) \sin \Theta d \Theta \\
& C_{y z}=\frac{\mu_{0}}{4 \pi} \int_{-R}^{R} L(\xi ; x, y, z) d \xi \\
& C_{z r}=\frac{\mu_{0}}{4 \pi} R \int_{0}^{2 \pi} B(\Theta ; x, y, z) \cos \Theta d \Theta \\
& +\frac{\mu_{0}}{4 \pi} \tan \delta \int_{-R}^{R} K(\xi ; x, y, z)[z+(R-\xi) \tan \delta] d \xi \\
& C_{z y}=\frac{\mu_{0}}{4 \pi} R \int_{0}^{2 \pi} B(\Theta ; x, y, z) \sin \Theta d \Theta \\
& C_{z z}=\frac{\mu_{0}}{4 \pi} \int_{-R}^{R} K(\xi ; x, y, z)[z+(R-\xi) \tan \delta] d \xi
\end{aligned}
$$




$$
B_{x, y}=\frac{\mu_{0}}{4} J_{x, y}\left[1+\frac{z}{\sqrt{z^{2}+R^{2}}}\right]
$$

and

$$
B_{z}=\frac{\mu_{0}}{2} J_{z}\left[\operatorname{sgn}(z)-\frac{z}{\sqrt{z^{2}+R^{2}}}\right]+\alpha(z)
$$

where $\quad \alpha(z)=\left\{\begin{array}{l}0 \text { outside the layer } \\ -\mu_{0} J_{z} \text { inside the layer. }\end{array}\right.$

The behavior of the $B_{i}$ s is controlled by the function $f(z)=$ $z / \sqrt{z^{2}+R^{2}}$, which is graphed in Figure 2. The vertical profile for each component is a smoothed ( $S$-shaped) step with inflection at the interface $(z=0)$. The step amplitude is $\mu_{0} / 2 J_{x, y}$ for $B_{x, y}$ and $-\mu_{0} J_{z}$ for $B_{z}$. We see from Figure 2 that the field values are 65 percent of their asymptotic values at a distance of $0.85 R$ from the interface, and 95 percent at $3 R$. We can define a $2 \sigma$ resolution of about $6 R$, beyond which the effects of the transition and the layer beyond the transition can be considered negligible.

The variation in the off-axis field as the transition is passed is significant. For this purpose, we have displayed horizontal cross-sections, i.e., maps, of the isofield values in the hole, more precisely of individual coefficients $C_{i j}$ (in units of $\mathrm{nT} / \mathrm{Am}^{-1}$ ). Field values can readily be computed for a given magnetization J. Figure 3 shows three sections at distances of $0.5,1$, and 2 (in units of $R$ ) from the interface. Both the intensities and the gradients decrease (in absolute value) away from the interface, as measured by $f(z)$ (Figure 2). A number of symmetries are obvious. At a given level, $C_{z z}$ has cylindrical symmetry with stronger gradients toward the edges of the hole; $C_{x x}$ and $C_{y y}$ have a similar geometry but with an elliptical shape for isolines, the short axis being along $O x$ and $O y$, respectively. $C_{x x}$ and $C_{y y}$ are deduced one from the other by a $\pi / 2$ rotation around $\mathrm{Oz} . C_{x y}$ and $C_{y x}$ have a weaker amplitude and hyperbolic isoline distribution. Off-diagonal terms with a $z$ index have stronger amplitudes

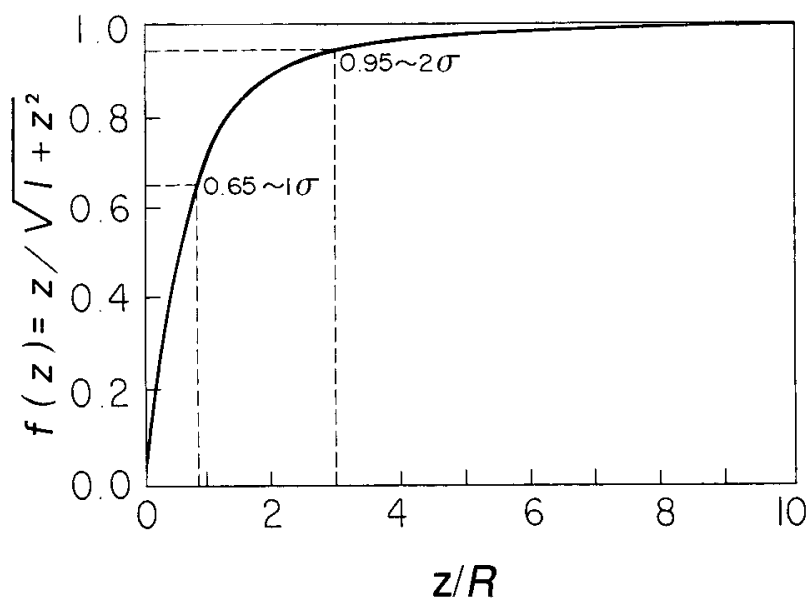

FIG. 2. Plot of the function $f(z)=z / \sqrt{1+z^{2}}$ which characterizes the evolution of magnetic field components across a magnetic polarity transition. The 65 percent and 95 percent levels can be used to define a $1 \sigma$ and $2 \sigma$ resolution thickness, normalized to the borehole radius $R$. and gradients and simple quasi-planar distributions, with elongation (strike) along the axis corresponding to the non-z, index. Altogether, there are four fundamental types of map distributions (i.e., symmetries): $\left(C_{x x}, C_{y y}\right),\left(C_{x y}, C_{y x}\right)$, $\left(C_{x z}, C_{y z}, C_{z y}, C_{z x}\right)$, and $\left(C_{z z}\right)$. When $z$ is changed into $-z$, coefficients $C_{x z}$ and $C_{z x}, C_{y z}$, and $C_{z y}$ remain the same, whereas $C_{x y}$ and $C_{y x}$ reverse in sign, and the diagonal terms reverse in shape with the addition of a constant term $100 \pi \mathrm{nT} / \mathrm{Am}^{-1}$ to $C_{x x}$ and $C_{y y}$ and $200 \pi \mathrm{nT} / \mathrm{Am}^{-1}$ to $C_{z z}$.

The coefficients mapped in Figure 3 can be combined to yield the full field for any $\mathbf{J}$ value. Two examples are given in Figure $4\left(D=20^{\circ}, I=60^{\circ}\right.$; and $D=45^{\circ}, I=35^{\circ}$; where $D$ is declination with respect to $\mathrm{Ox}$ and $I$ is inclination with respect to $\mathrm{Oxy}$ ) for a distance $R$ on either side of the interface. Roughly speaking, the center of the hole is a center of symmetry. In addition, the horizontal projection of $\mathbf{J}$ is an axis of symmetry at any given level for the vertical component. We find that declination can be recovered easily from the $B_{z}$ maps. The distance of the extremum of $B_{z}$ from the center of the hole varies regularly with inclination, being 0 for a vertical magnetization and $R$ for a horizontal one.

Effects of dipping layers.-The full equations given in the Appendices and in Table 1 have been used to calculate the effects of dipping layers. The $C_{i j}$ are presented in Figure 5, in the same format as in Figure 3. A single distance from the interface, chosen here to be $R$, is sufficient to illustrate pattern changes; configurations both above and below this interface are given to show how the symmetries are broken. A dip of $20^{\circ}$ in the direction of $O x$ has been selected. The basic configuration of Figure 3 (i.e., dip $=0^{\circ}$ ) is slightly distorted as a sort of rotation around Oy. Ox remains the only symmetry (or antisymmetry) axis. The sense and amount of offset of key symmetry elements of the zero-dip patterns are roughly the same for all $C_{i j}$ at a given level; the sense is reversed when the transition is crossed. Because of the dip, the total variation of $C_{x x}$ over the hole at a given level tends to be increased at the expense of $C_{z z}$ compared to the case of zero dip (see also below and Figure 7). The offset of the maxima of diagonal coefficients $C_{i i}$ is the same for all three $C_{i i}$ at a given level, and increases with dip. For a given dip, the maxima offset decreases with distance from the interface. This is shown by Figure 6, in which we have plotted the normalized offset with respect to distance from the interface for various values of dip ranging from $10^{\circ}$ to $50^{\circ}$ (offsets are of course identically 0 for zero dip). Beyond the previously defined resolution distance of $3 R$, offsets are noticeable (i.e., larger than say $0.1 R$ ) only when the dip reaches or exceeds $20^{\circ}$. Again, only measurements sufficiently close to the interface can provide quantitative information when there is dip.

We have computed vertical axial profiles of field components, in a slightly more complex case with four transitions and five layers, with magnetization $\mathbf{J}=(1,0,1)$ and $-\mathbf{J}$ alternately (Figure 7$)$. Three values of $\operatorname{dip}\left(0^{\circ}, 10^{\circ}, 20^{\circ}\right)$ have been selected. The peak-to-trough amplitude of $B_{x}$ increases at the expense of that of $B_{z}$ as the dip increases. The $\Delta B_{x} / \Delta B_{z}$ ratio changes from 0.5 to 2 when the dip increases from 0 to $20^{\circ}$. Figure 7 also provides additional indications on the resolving power of the method. 

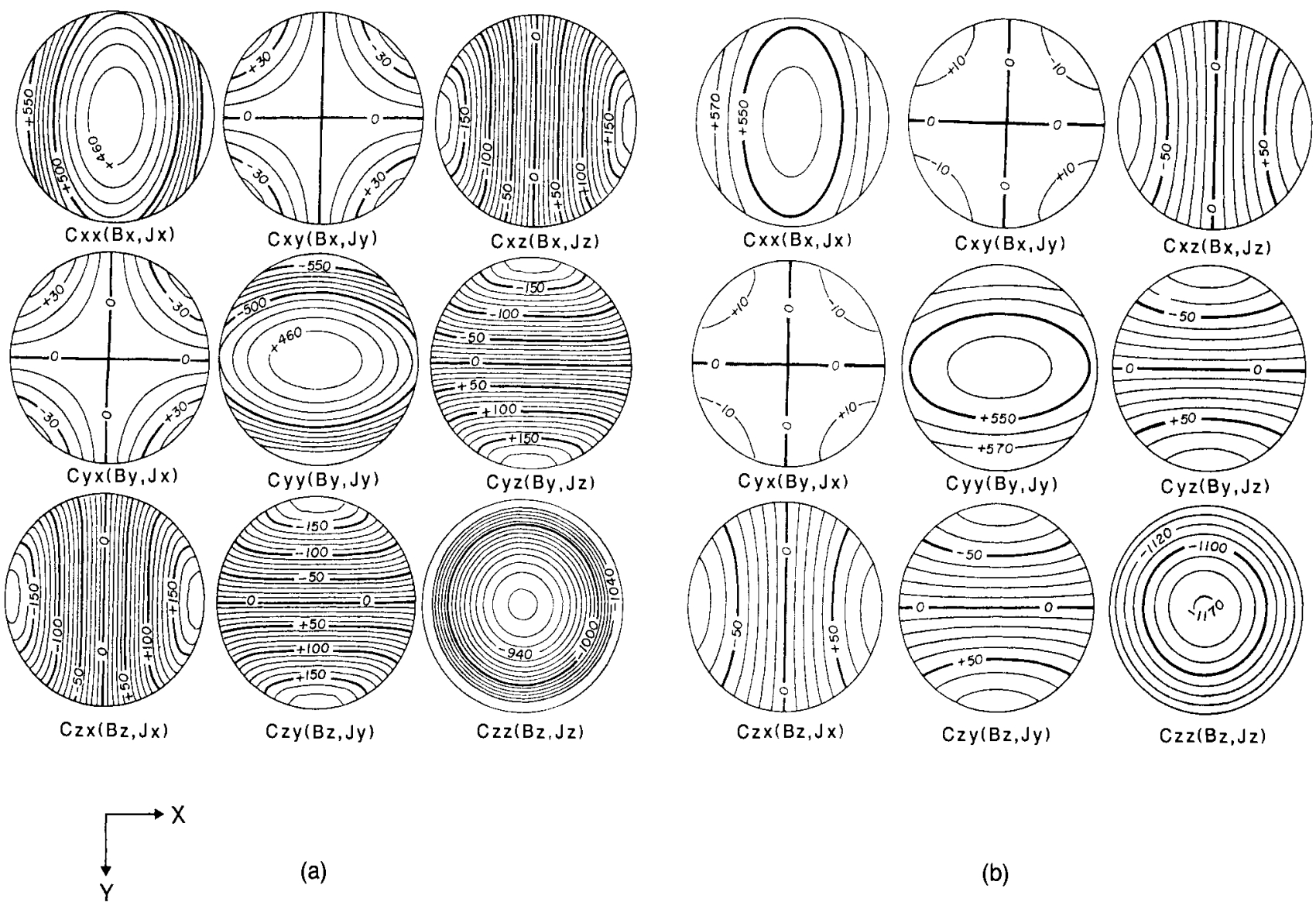

(a)
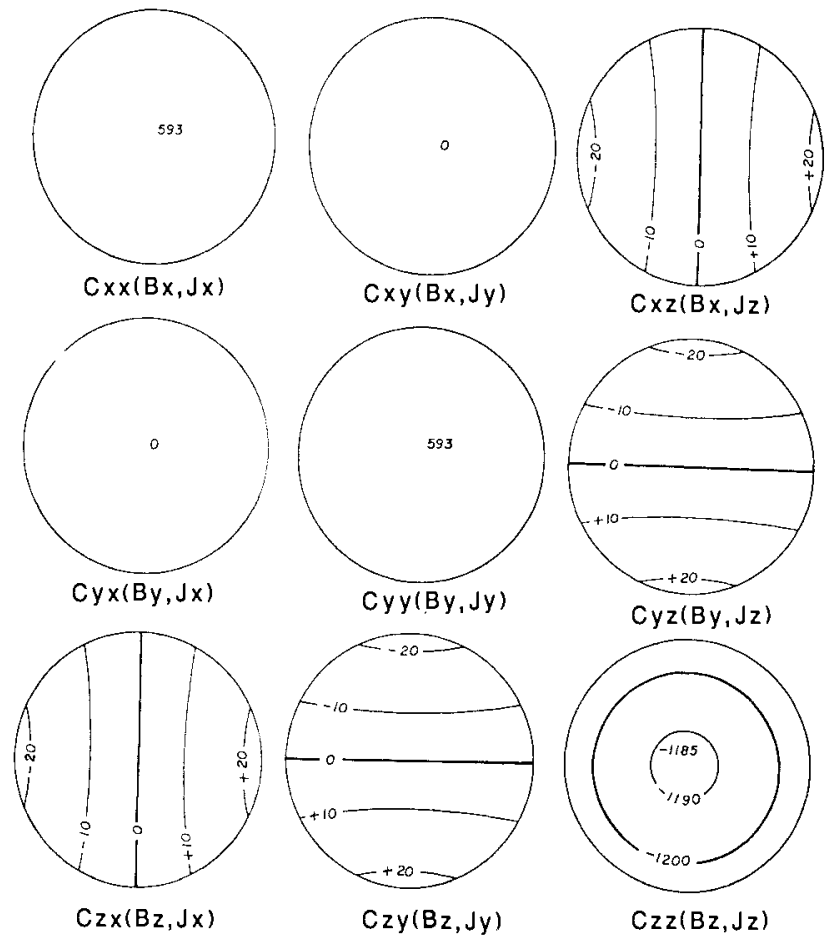

(c)

FIG. 3. The magnetic field as a function of measurement position within a borehole at three different levels below the top of a uniformly magnetized semiinfinite layer. (a) $z=0.5 R$, (b) $z=1 R$, (c) $z=2 R$. The contoured maps show the nine coefficients of the tensor $\mathbf{C}$ which relates the field $\mathbf{B}$ to the magnetization $\mathbf{J}\left(B_{i}=C_{i j} J_{j}\right.$ summed over $\left.j\right)$. Units are $\mathrm{nT} / \mathrm{Am}^{-1}$. 


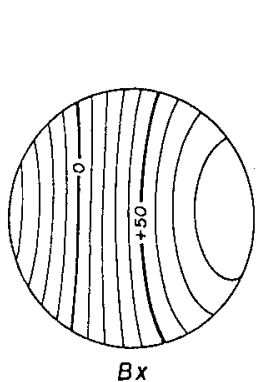

$Z=-1 R$

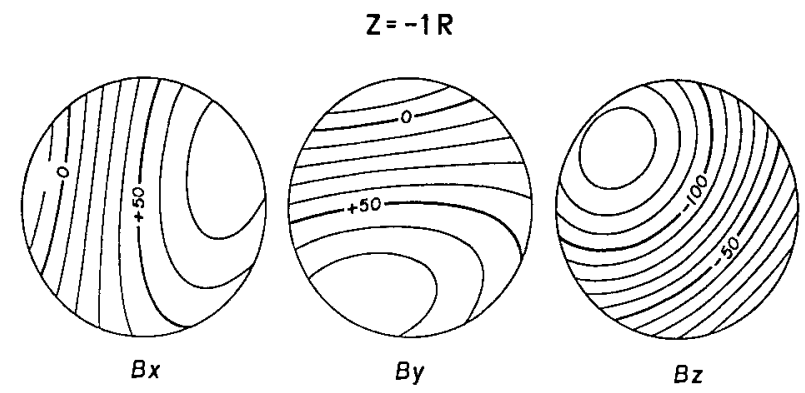

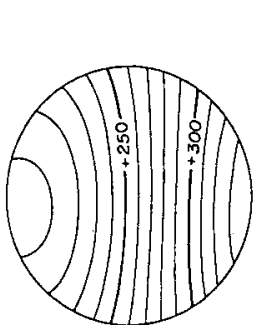

$B x$

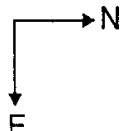

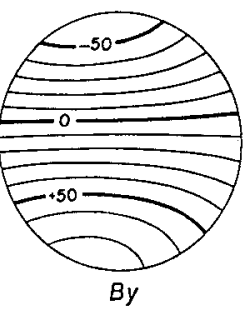

$Z=1 R$

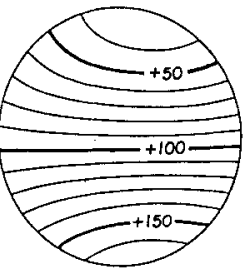

By

(a)
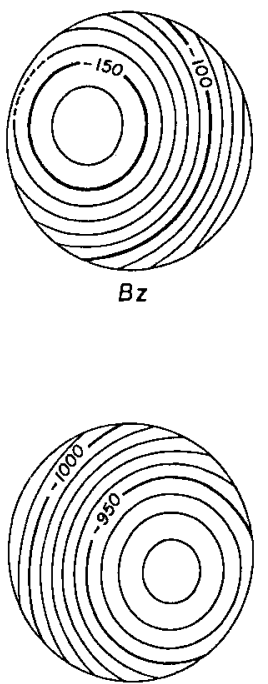

$B z$

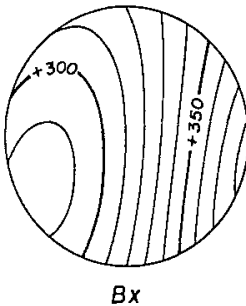

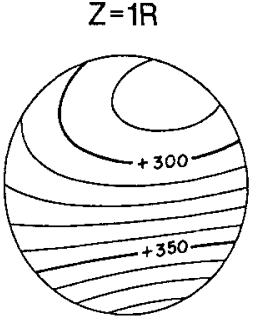

By

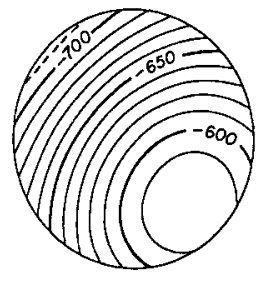

$B z$

Fig. 4. Horizontal maps of the three remanent field components in a borehole at two levels $Z= \pm R$ for two directions of magnetization: (a) $D=20^{\circ}, I=60^{\circ}$; (b) $D=45^{\circ}, I=35^{\circ}$. The model used is the same as in the previous figure. Units are $\mathrm{nT} / \mathrm{Am}^{-1}$.
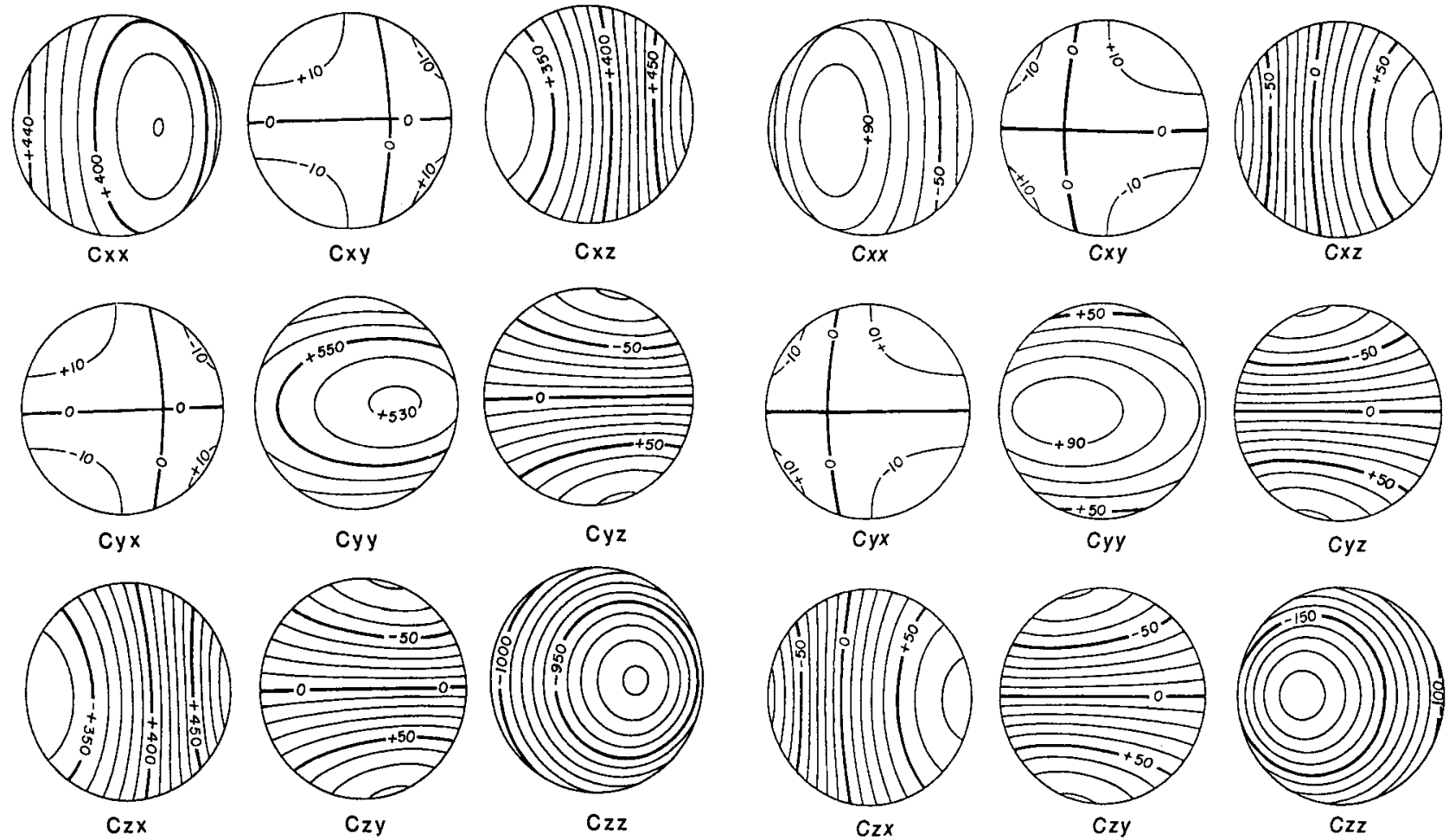

(a)

(b)

FIG. 5. The nine tensor coefficients within a borehole for a dip $\delta$ of $20^{\circ}$. The sense of offset of the patterns reverses as one moves from above ( $+R$; case a) to below $(-R$; case b) the upper interface. The offset of the pattern increases with dip. 


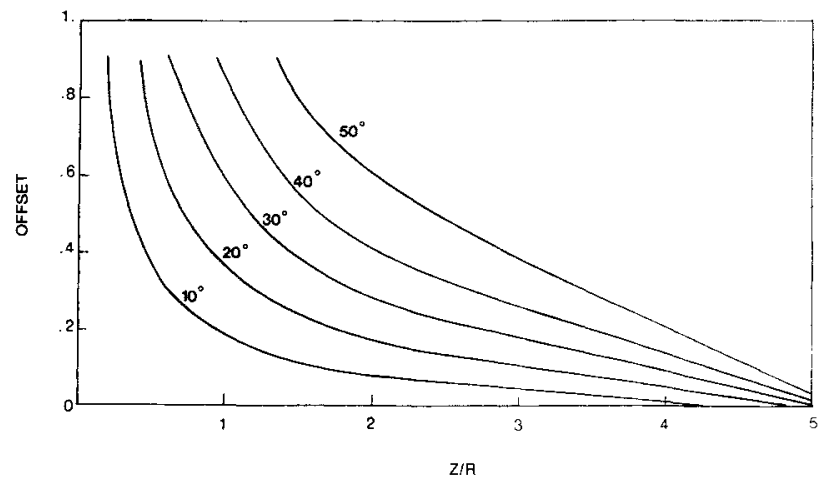

FIG. 6. Decrease of the offset of the diagonal field coefficients with distance from the interface using different dips. Units on both axes are hole radius $R$.
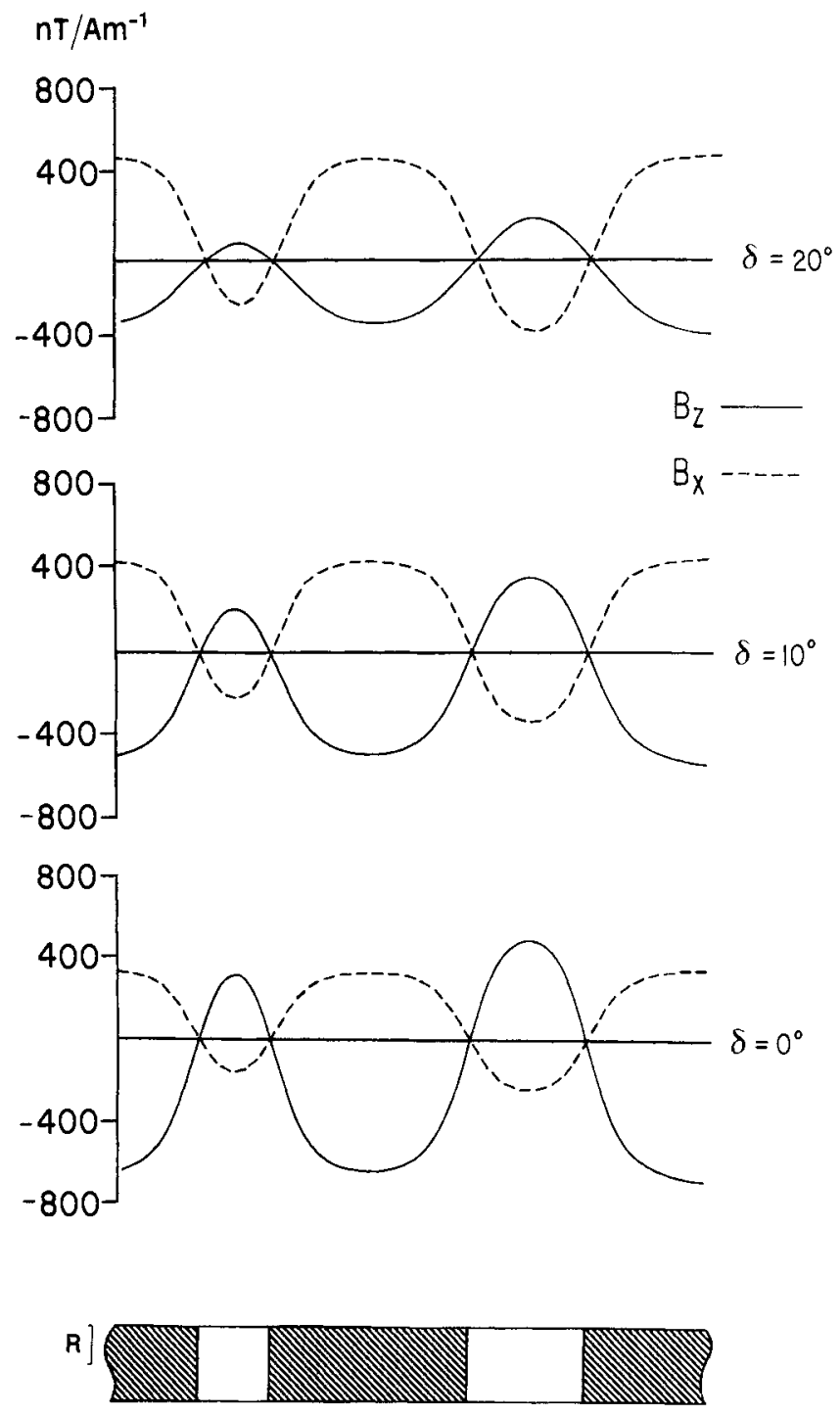

Fig. 7. Simulated axial profiles of $B_{x}$ and $B_{z}$ components for a configuration of five drilled layers with opposite magnetizations $\pm \mathbf{J}(1,0,1)$. As the dip $\delta$ increases, the relative amplitudes of the components change.
Combined effects of dip and magnetization directions. - It is clear from an inspection of Figures 4 and 5 that magnetization direction (characterized by $D$ and $I$ ) and bedding attitude (characterized by strike $s$ and dip $\delta$ ) combine their effects in distorting the patterns of Figure 3, offsetting the extrema positions and altering the symmetry patterns. For instance, we have determined the variation of offset of the extrema of $B_{x}$ and $B_{z}$ as a function of $\delta$ and $I$ (and distance from the interface) when $D=0^{\circ}$ and dip is only along $O x$. In that case, Ox remains a symmetry axis on which the extrema are located. For $B_{-}, I$ and $\delta$ add their effects (Figure 8), whereas they act against each other in $B_{x}$ (not shown in Figure 8).

Effect of nonuniform dip.-The same formalism can in principle also be used when the dip of the layers is not uniform and changes with depth. Layers are not of infinite extent in all directions and the solution is not rigorously correct. However, if differences in dip are small enough that layers intersect far from the hole, we can use the above formalism as an approximation. This approximation appears to be acceptable (i.e., within 10 percent of the exact solution) when the layer interface intersection is farther than about 10 times its thickness away from the hole. It results in the introduction of a long-wavelength downhole trend in the magnetic components and a modification of the amplitude ratios such as $\Delta B_{x} / \Delta B_{z}$ (Figure 9).

\section{DISCUSSION}

In the previous sections, we have outlined the magnetic field distribution within a hole bored through a sequence of layers with varying magnetizations, when these layers have plane, possibly dipping, interfaces, with an emphasis on changes in magnetic induction close to polarity transitions. Results have been presented both in terms of axial vertical

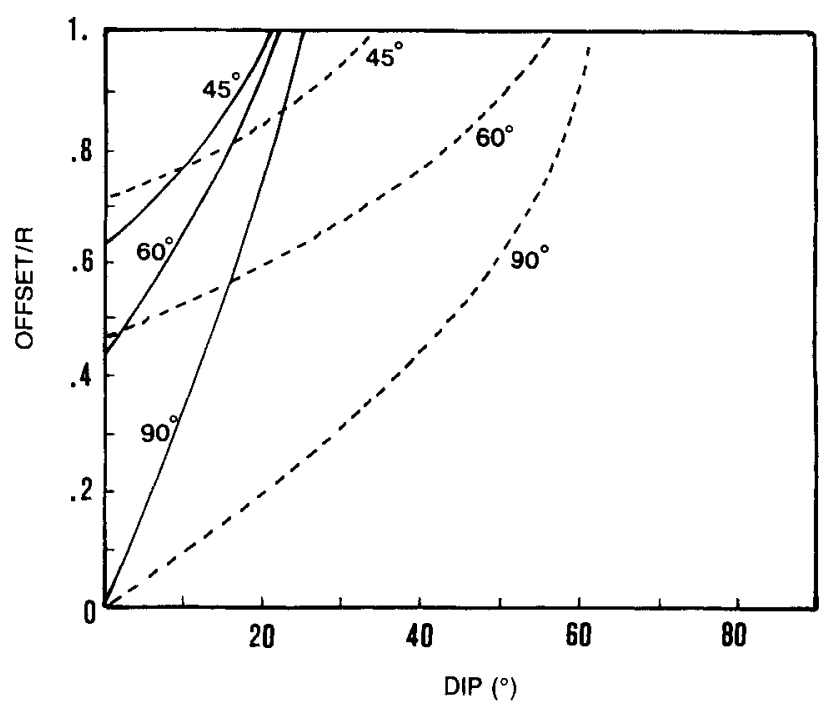

FIG. 8. Change with the dip of the offset of the vertical $\left(B_{z}\right)$ component due to the joint effects of dip and magnetic inclination of the layer. The dashed and solid lines correspond to levels $z=2 R$ and $1 R$, respectively, for three magnetic inclinations $I=45^{\circ}, 60^{\circ}$, and $90^{\circ}$. 
profiles and of maps of sections of the hole at various distances from the interfaces. This has led us to define a resolution thickness within which most ( 95 percent) of the magnetic changes related to a polarity interval take place. This resolution is about six times the radius of the hole, i.e., on the order of $1 \mathrm{~m}$ for a typical hole. The corresponding time resolution in a magnetostratigraphic analysis will, of course, depend on sedimentation rate. For a rate of $10 \mathrm{~m} / \mathrm{Myr}$, the time resolution is on the order of $10^{5} \mathrm{yr}$,
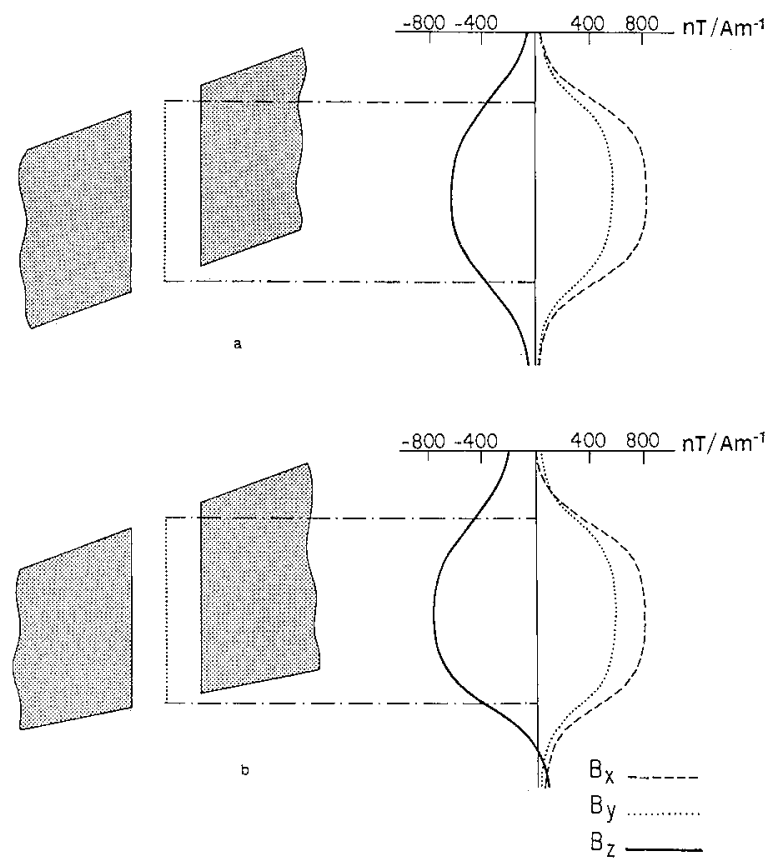

FIG. 9. Profiles of the three field components across a magnetized $\mathbf{J}(1,0,1)$ layer for two configurations of dips (in the Ox direction: (a) uniform dip of $20^{\circ}$, (b) different dips between the upper $\left(20^{\circ}\right)$ and the lower $\left(10^{\circ}\right)$ interface.
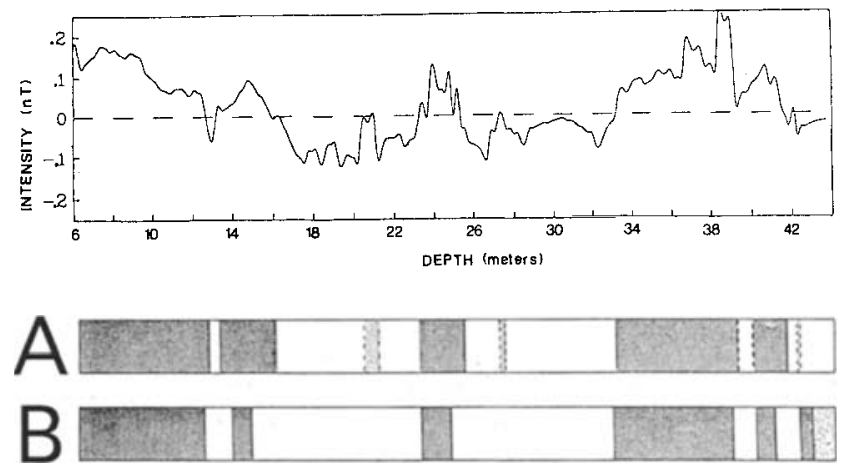

FIG. 10. Calculated axial magnetic anomaly profile along the DSDP 573A borehole (Weinrich and Theyer, 1985). This pattern is constructed from sample NRM, i.e., each sample defines a layer with a characteristic magnetization. The field is projected onto the present geomagnetic direction $\left(D=-3^{\circ}\right.$ and $I=21.5^{\circ}$ ). The polarity intervals which can be deduced from this calculated profile are shown in column $\mathrm{A}$; the dashed lines indicate uncertain identifications. The "true" polarity intervals, obtained after onboard demagnetization of the original samples (Weinrich and Theyer, 1985), are presented in column B for comparison. which tends to be smaller than the length of many polarity chrons, particularly for ages older than $10 \mathrm{Myr}$ (e.g., Cox, 1982).

A parameter also related to resolution is the intensity of the natural remanent magnetization (NRM) of the rocks. The most magnetized rocks are mafic lavas, which generally bear a thermal remanent magnetization of order $10^{-1} \mathrm{Am}^{-1}$. Mafic lavas generate fields of hundreds of $n T$, which are readily identified with the crudest magnetometers. Volcanic flows would constitute conspicuous markers in a borehole. They are a central focus of some Ocean Drilling Project crustal holes (Ponomarev and Nechoroshkov, 1983, 1984; Parker and Daniell, 1979; Pariso and Johnson, 1988). Red sandstones and siltstones carrying hematite are often fairly strongly magnetized, typically in the range $10^{-2}$ to $10^{-3}$ $\mathrm{Am}^{-1}$. Limestones tend to be much weaker, from $10^{-3}$ $\mathrm{Am}^{-1}$ down to $10^{-5} \mathrm{Am}^{-1}$ (see e.g., Lowrie and Heller, 1982) and below $\left(10^{-5} \mathrm{Am}^{-1}\right.$ is often the sensitivity level of cryogenic magnetometers used in paleomagnetic laboratories). For a value of $10^{-3} \mathrm{Am}^{-1}$, i.e., in the lower range for red beds and in the upper one for limestones, the anomalies to be detected are on the order of several $10^{-1} \mathrm{nT}$. Provided that a number of precautions are observed, sensitive fluxgate magnetometers are available for magnetostratigraphic application.

To illustrate the problems of depth resolution and downhole variations of the magnetization intensity, we have calculated a synthetic axial profile of the anomalous field along ODP hole 573A based on NRM data from Weinrich and Theyer (1985). Each sample is assumed to represent a flat homogeneous layer extending from the sample depth to the midpoint of the two adjoining samples. The peak-to-peak intensity is about $0.4 \mathrm{nT}$ (Figure 10). Despite many short-wavelength oscillations due to intensity fluctuations within layers of a given magnetic polarity, the main polarity changes are located on the total field anomaly profile (provided an instrumental resolution better than $0.1 \mathrm{nT}$ is used). A possible magnetostratigraphic interpretation is shown in column A. Possible reversals corresponding to significant amplitude changes, rather than true reversals, are indicated at depths of 20,27 , and $39 \mathrm{~m}$. The samples have actually been demagnetized and their characteristic primary magnetization isolated (Weinrich and Theyer, 1985), providing an accurate magnetostratigraphy (column B). We find a very reasonable match between the logs deduced from classical paleomagnetism and downhole magnetic field distribution. The events at 20 and $27 \mathrm{~m}$ appear to be due to intensity changes within intervals of constant polarity, whereas that at $39 \mathrm{~m}$ appears to be due to reversal. In this example, correlation with the reversal time scale and absolute dating may be achieved.

The maps in Figures 3 to 5 show that very significant information is contained within a given level when a polarity interface is approached. If the three components could be surveyed (in the classical sense of magnetic surveying), information on the direction of magnetization and on the dip of the interface could be recovered. These two sources of distortion are superimposed (Figure 6), but external constraints could be used to separate them: for instance, the magnetization direction could be recovered if the dip were independently evaluated. The location of extrema or symmetry patterns in the maps, as well as the detection of gradients typically stronger near the edges of the hole, would therefore be greatly facilitated 
by sufficiently dense surveying. There is a tradeoff between the requirement to install as many triaxial (vector) sensors as possible at a given horizontal level in the tool and the space actually available for the tool, given the attainable level of miniaturization of the magnetic sensors. It would seem desirable as a minimum to have several sensors off-axis, for instance in a cross shape. With both downward displacements and rotation around the hole axis, rather dense measurements could be made and gradients of all components evaluated.

The simple modeling method developed here demonstrates several aspects relevant to the feasibility of downhole magnetostratigraphy. It provides guidelines for the design of a multisensor vector probe which could, in favorable cases, identify sequences of magnetic polarity transitions. The weak magnetization of most sedimentary rocks would require that the most sensitive magnetometers be used, in a differential mode with a surface station in order to reduce transient and induced magnetic fields. Although most practicing paleomagnetists would argue that definitive magnetostratigraphy requires complete and careful demagnetization of samples in the laboratory and proper identification of remanence-carrying phases, the example in Figure 10 shows that in some cases the uncleaned NRM could be sufficient to reveal the polarity sequence, if not the accurate remanence directions.

\section{ACKNOWLEDGMENTS}

We thank A. Galdeano for many discussions and for providing the drafting routines and D. Vandamme, R. Enkin, and Y. Gaudemer for discussions and encouragement. We are particularly grateful to G. Dupin for the final drafting and to the anonymous reviewers for careful and constructive comments. IPGP contribution NS 1064.

\section{REFERENCES}

Clerc, G., Frignet, B., and Tabbagh, 1983, Transmitter-receiver induction techniques and probes developed for surface and borehole measurements: Application to archaeology and mineral exploration: J. Geomag. Geoelect., 35, 443-454.

Courtillot, V., and Le Mouel, J. L., 1988, Time variations of the Earth's magnetic field: From daily to secular: Ann. Rev. Earth Planet. Sci., 16, 389-476.

Cox, A. V., 1982, A geological time scale, in Harland, W. B. Cox, A. V., Llewellyn, P. G., Pickton, C. A. G., Smith, A. G., and Walters, R., Eds., A geological time scale: Cambridge Univ. Press, 63-84.

Lowrie, W., and Heller, F., 1982, Magnetic properties of marine limestones: Rev. Geophys. Space Phys., 20, 171-193.

McElhinny, M. W., 1973, Paleomagnetism and plate tectonics: Cambridge Univ. Press.

Pariso, J., and Johnson, H. P., 1988, A downhole magnetic logging tool for the Ocean Drilling Program: EOS, 69, 818 .

Parker, R. L., and Daniell, G. J., 1979, Interpretation of borehole magnetometer data: J. Geophys. Res., 84, 5467-5479.

Ponomarev, V. N., and Nechoroshkov, V. L., 1983, First measurements of the magnetic field within the ocean crust: Deep Sea Drilling Project Legs 68 and 69: Init. Repts. DSDP, 69, 271-279.

1984. Downhole magnetic measurements in oceanic crustal hole $395 \mathrm{~A}$ on the mid-Atlantic ridge: Init. Repts. DSDP, 78B, 731-739.

Pozzi, J. P., Martin, J. P., Pocachard, J., Feinberg, H., and Galdeano, A., 1988, In-situ magnetostratigraphy: interpretation of magnetic logging in sediments: Earth Planet. Sci. Lett., 88, $357-373$.

Rochette, P., 1988, La susceptibilité anisotrope des roches faiblement magnétiques, origines et applications: Doctoral thesis, Univ. of Grenoble.

Tauxe, L., Butler, R., and Herguera, J. C., 1987, Magnetostratigraphy: in pursuit of missing links: Rev. Geophys. Space Phys., 25, 939-950.

Weinrich, N., and Theyer, F., 1985, Paleomagnetism of Deep Sea Drilling Project Leg 85 sediments: Neogene magnetostratigraphy and tectonic history of the central equatorial Pacific: Init. Repts. DSDP, 85, 849-901

Zlotnicki, J., 1986, Magnetic measurements on La Soufrière Volcano (Lesser Antilles), 1976-1984: A re-examination of the volcanomagnetic effects observed during the volcanic crisis of 1976 1977: J. Volc. Geotherm. Res., 30, 80-116.

\section{APPENDIX A}

\section{FIELD COEFFICIENTS: LATÉRAL SURFACE OF CYLINDER}

In this appendix, we calculate the field coefficients for the lateral surface of a semiinfinite cylinder with an inclined superior surface (Figure 1a)

We determine the magnetostatic potential at point $P(x, y, z)$ by integrating $d V(P)=\left(\mu_{0} / 4 \pi\right)(d q / r) ; r$ is the distance between $P$ and point $Q(\xi=R \cos \Theta, \eta=R \sin \Theta, \tau)$, where $H(\Theta)=2 R \tan \delta \sin ^{2}(\Theta / 2) \leqslant \tau<\infty$ and $\delta$ is the dip of the superior surface (Figure 1a). $d q=\mathbf{J} \cdot \mathbf{n} d S$ is the equivalent magnetic charge on $d S=R d \tau d \Theta$.

We obtain

$$
V(P)=\frac{\mu_{0}}{4 \pi} \int_{0}^{2 \pi} \mathbf{J} \cdot \mathbf{n}(\Theta) \int_{H(\Theta)}^{\infty} \frac{d \tau}{\sqrt{(x-R \cos \Theta)^{2}+(y-R \sin \Theta)^{2}+(z-\tau)^{2}}} d \Theta .
$$

Using

$$
\begin{aligned}
A(\Theta ; x, y, z)= & \int_{H(\Theta)}^{\infty} \frac{d \tau}{\left[(x-R \cos \Theta)^{2}+(y-R \sin \Theta)^{2}+(z-\tau)^{2}\right]^{3 / 2}} \\
= & \frac{1}{(x-R \cos \Theta)^{2}+(y-R \sin \Theta)^{2}} \\
& \times\left[1+\frac{2 R \tan \delta \sin ^{2}(\Theta / 2)+z}{\sqrt{(x-R \cos \Theta)^{2}+(y-R \sin \Theta)^{2}+\left[z+2 R \tan \delta \sin ^{2}(\Theta / 2)\right]^{2}}}\right]
\end{aligned}
$$




$$
\begin{aligned}
B(\Theta ; x, y, z) & =\int_{H(\Theta)}^{\infty} \frac{(z-\tau) d \tau}{\left[(x-R \cos \Theta)^{2}+(y-R \sin \Theta)^{2}+(z-\tau)^{2}\right]^{3 / 2}} \\
& =-\frac{1}{\sqrt{(x-R \cos \Theta)^{2}+(y-R \sin \Theta)^{2}+\left[z+2 R \tan \delta \sin ^{2}(\Theta / 2)\right]^{2}}},
\end{aligned}
$$

we find

$$
\begin{aligned}
& B_{x x}=-\frac{\partial V\left(J=J_{x}\right)}{\partial x}=\frac{\mu_{0}}{4 \pi} J_{x} R \int_{0}^{2 \pi} A(\Theta ; x, y, z)(x-R \cos \Theta) \cos \Theta d \Theta, \\
& B_{x y}=-\frac{\partial V\left(J=J_{y}\right)}{\partial x}=\frac{\mu_{0}}{4 \pi} J_{y} R \int_{0}^{2 \pi} A(\Theta ; x, y, z)(x-R \cos \Theta) \sin \Theta d \Theta, \\
& B_{y x}=-\frac{\partial V\left(J=J_{x}\right)}{\partial y}=\frac{\mu_{0}}{4 \pi} J_{x} R \int_{0}^{2 \pi} A(\Theta ; x, y, z)(y-R \sin \Theta) \cos \Theta d \Theta, \\
& B_{y y}=-\frac{\partial V\left(J=J_{y}\right)}{\partial y}=\frac{\mu_{0}}{4 \pi} J_{y} R \int_{0}^{2 \pi} A(\Theta ; x, y, z)(y-R \sin \Theta) \sin \Theta d \Theta, \\
& B_{z x}=-\frac{\partial V\left(J=J_{x}\right)}{\partial z}=\frac{\mu_{0}}{4 \pi} J_{x} R \int_{0}^{2 \pi} B(\Theta ; x, y, z) \cos \Theta d \Theta,
\end{aligned}
$$

and

$$
\begin{gathered}
B_{z y}=-\frac{\partial V\left(J=J_{y}\right)}{\partial z}=\frac{\mu_{0}}{4 \pi} J_{y} R \int_{0}^{2 \pi} B(\Theta ; x, y, z) \sin \Theta d \Theta . \\
\text { APPENDIX B } \\
\text { FIELD COEFFICIENTS: INCLINED ELLIPSE }
\end{gathered}
$$

Here we calculate the field components created by an inclined ellipse with dip $\delta$ (Figure $1 b$ ).

The magnetostatic potential at point $P(x, y, z)$ is given by integrating $d V(P)=\left(\mu_{0} / 4 \pi\right)(d q / r)$, where $d q=\mathbf{J} \cdot \mathbf{n}(d \xi d \eta / \cos \delta)$. The geometry is shown is Figure $1 \mathrm{~b}$. We have $\mathbf{J} \cdot \mathbf{n}=J_{x} \sin \delta-J_{z} \cos \delta$. By integrating along bands parallel to the Oy axis, we obtain (with $C(\xi)=\sqrt{R^{2}+\xi^{2}}$ ):

$$
V(P)=\frac{\mu_{0}}{4 \pi} \frac{J_{x} \sin \delta-J_{z} \cos \delta}{\cos \delta} \int_{-R}^{R} \int_{-C(\xi)}^{C(\xi)} \frac{d \xi d \eta}{\sqrt{(x-\xi)^{2}+(y-\eta)^{2}+[z+(R-\xi) \tan \delta]^{2}}} .
$$

Given

$$
\begin{aligned}
K(\xi ; x, y, z)= & \int_{-C(\xi)}^{C(\xi)} \frac{d \eta}{\left\{(x-\xi)^{2}+(y-\eta)^{2}+[z+(R-\xi) \tan \delta]^{2}\right\}^{3 / 2}} \\
= & \frac{1}{(x-\xi)^{2}+[z+(R-\xi) \tan \delta]^{2}}\left[\frac{\sqrt{R^{2}-\xi^{2}}-y}{\sqrt{(x-\xi)^{2}+\left(y-\sqrt{R^{2}-\xi^{2}}\right)^{2}+[z+(R-\xi) \tan \delta]^{2}}}\right. \\
& \left.+\frac{\sqrt{R^{2}-\xi^{2}}+y}{\sqrt{(x-\xi)^{2}+\left(y+\sqrt{\left.R^{2}-\xi^{2}\right)^{2}+[z+(R-\xi) \tan \delta]^{2}}\right.}}\right]
\end{aligned}
$$

and 


$$
\begin{aligned}
L(\xi ; x, y, z) & =\int_{-C(\xi)}^{C(\xi)} \frac{(y-\eta) d \eta}{\left\{(x-\xi)^{2}+(y-\eta)^{2}+[z+(R-\xi) \tan \delta]^{2}\right\}^{3 / 2}} \\
& =\frac{1}{\sqrt{(x-\xi)^{2}+\left(y-\sqrt{R^{2}-\xi^{2}}\right)^{2}+[z+(R-\xi) \tan \delta]^{2}}} \\
& -\frac{1}{\sqrt{(x-\xi)^{2}+\left(y+\sqrt{R^{2}-\xi^{2}}\right)^{2}+[z+(R-\xi) \tan \delta]^{2}}}
\end{aligned}
$$

we find

$$
\begin{aligned}
& B_{x}=-\frac{\partial V}{\partial x}=\frac{\mu_{0}}{4 \pi} \frac{J_{x} \sin \delta-J_{z} \cos \delta}{\cos \delta} \int_{-R}^{R} K(\xi ; x, y, z)(x-\xi) d \xi, \\
& B_{y}=-\frac{\partial V}{\partial y}=\frac{\mu_{0}}{4 \pi} \frac{J_{x} \sin \delta-J_{z} \cos \delta}{\cos \delta} \int_{-R}^{R} L(\xi ; x, y, z) d \xi,
\end{aligned}
$$

and

$$
B_{z}=-\frac{\partial V}{\partial z}=\frac{\mu_{0}}{4 \pi} \frac{J_{x} \sin \delta-J_{z} \cos \delta}{\cos \delta} \int_{-R}^{R} K(\xi ; x, y, z)[z+(R-\xi) \tan \delta] d \xi .
$$

